\title{
Communication Patterns for Expressing Real-Time Requirements Using MSC and Their Application to Testing
}

\author{
Helmut Neukirchen ${ }^{1}$, Zhen Ru Dai ${ }^{2}$, and Jens Grabowski ${ }^{1}$ \\ 1 Institute for Informatics, University of Göttingen \\ Lotzestr. 16-18, D-37083 Göttingen, Germany \\ \{neukirchen, grabowski\}@informatik. uni-goettingen.de \\ ${ }^{2}$ Fraunhofer FOKUS, Competence Center TIP \\ Kaiserin-Augusta-Allee 31, D-10589 Berlin, Germany \\ dai@fokus.fraunhofer.de
}

\begin{abstract}
This paper introduces real-time communication patterns (RTC-patterns) for capturing real-time requirements of communication systems. RTC-patterns for some of the most common real-time requirements are presented. They are formalized by using Message Sequence Charts (MSCs). The application of RTC-patterns to testing is explained by an example. The example shows how real-time requirements which are expressed using RTC-patterns can be related to TIMEDTTCN-3 evaluation functions.
\end{abstract}

\section{Introduction}

The motivation for the work presented in this paper comes from our research on test specification and test generation for testing real-time requirements of communication systems. Especially, we investigate graphical specification methods that can be used in all phases of an integrated system development methodology and that allow an automated generation and implementation of test cases.

We use the Testing and Test Control Notation (TTCN-3) [5] as test implementation language and developed TIMEDTTCN-3 [3] as an associated real-time extension to support the test of real-time requirements. For graphical test specification, we apply the Message Sequence Chart (MSC11) language [13]. The MSCbased specification of real-time test cases and generation of TIMEDTTCN-3 code from MSC test specifications is explained in [4].

Even though it is possible to generate TIMEDTTCN-3 code automatically for each MSC test description, we would like to facilitate and harmonize the use of TIMEDTTCN-3 by providing a common set of test evaluation functions. This would make test results more comparable and avoid misinterpretations due to the use of different or erroneous evaluation functions. The key issue of this

\footnotetext{
${ }^{1}$ The term $M S C$ is used both for a diagram written in the MSC language and for the language itself.
} 
approach is the identification of commonly applicable evaluation functions for TIMEDTTCN-3 test cases. Such functions are used to evaluate relations among time stamps of events, which are observed during a test run. An evaluation function is related to the number of interfaces of the system under test, the number of time stamps to be considered and the number of relations among these time stamps. It would be necessary to provide an infinite set of evaluation functions to cover all cases. This is not possible and, therefore, we look for a mechanism to identify evaluation functions for the most common cases.

Our idea is to use real-time communication patterns (RTC-patterns) for expressing real-time requirements and to provide evaluation functions for these patterns only. By using RTC-patterns during test design or by scanning test specifications for RTC-patterns, it is possible to use predefined evaluation functions in TIMEDTTCN-3 test descriptions.

The idea of patterns is not new. Software patterns as described in 62 ] focus on structural aspects of software design. Conventional software patterns are independent of an implementation language and described in a rather informal manner. Different from software patterns, SDL patterns [7] are tailored to the development of SDL 12 systems. They benefit from the formal SDL semantics, which offers the possibility of precisely specifying how to apply a specific pattern, under which assumptions this will be allowed, and what properties result for the embedding context.

RTC-patterns are used to describe real-time requirements in form of time relations among communication operations at the interfaces of a communication system. We use MSC for the pattern description. The formality of MSC allows formalizing at least some parts of the pattern instantiation. Even though the application domain of testing communication systems motivates our work on RTC-patterns, we believe that such patterns are of general interest for system development. Therefore, we present RTC-patterns independent of the testing domain (Section 2) and explain afterwards their application to testing (Section 3).

\section{MSC and Patterns}

This section gives a short introduction into the subset of the MSC language, which is used in this paper, and presents MSC patterns for capturing real-time requirements.

\section{$2.1 \mathrm{MSC}$}

Basically, an MSC describes the flow of messages between the instances of a communication system. For example, the MSC Referenced (Fig. 11 ) includes three instances, i.e., PCO, System ${ }_{1}$ and System $_{2}$, and specifies that message $\mathrm{m} 3$ is sent from System 1 to System .

The MSC language supports abstraction from and refinement of behavior by decomposed instances and references. The decomposition mechanism allows to refine the behavior of an instance. This is shown in Fig. 17a and 1p. The keywords 


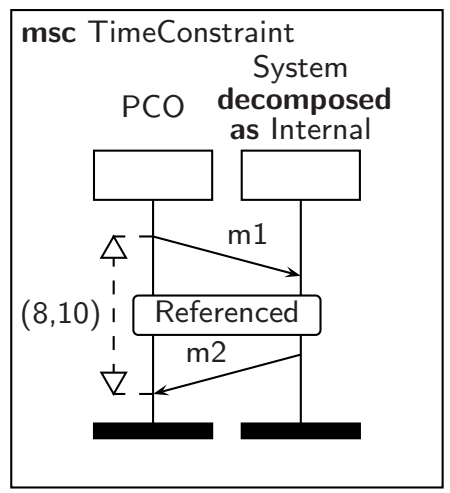

(a) Top Level MSC

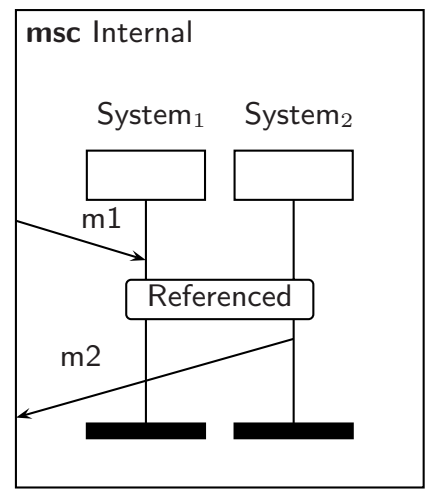

(b) Decomposition of System

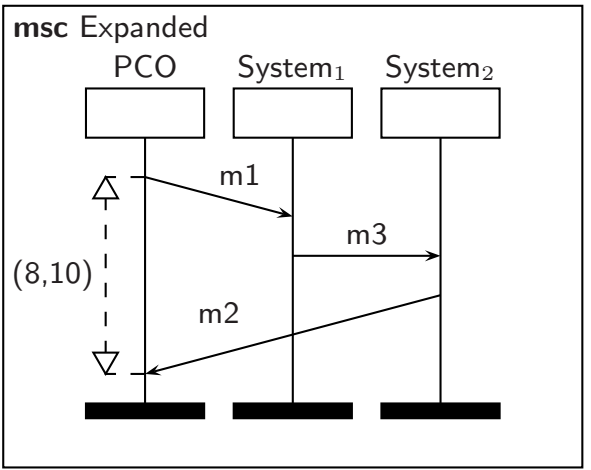

(d) Expansion of MSC (a) using (b) and (c)

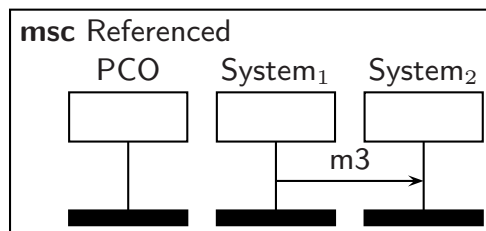

(c) MSC referenced in (a)

Fig. 1. Used MSC Constructs

decomposed as followed by the name Internal in the header of instance System (Fig. 19) indicates that System is an abstraction of the behavior specified by MSC Internal (Fig. 1b). The MSCs in Fig. 1a and 1b also contain reference symbols, which both refer to the MSC Referenced. The semantics of a reference symbol is given by the referenced MSC, i.e., the behavior of the referenced MSC replaces the reference. By applying the rules for decomposed instances and references, the MSC TimeConstraint can be expanded to the MSC shown in Fig. 11d.

For the specification of complex communication behavior in a compact manner within one diagram, MSC provides inline expressions. In this paper, we only use loop inline expressions to specify the repeated occurrence of events. Fig. 4 presents an example, the behavior of the reference symbols loopedPreamble, ResponseTimePattern and loopedPostamble is repeated $n$ times.

MSC allows to attach time annotations to events like sending or receiving a message. In this paper we make use of relative time constraints which limit the duration between two events. A time constraint is shown in Fig. 17a: the time difference between sending $\mathrm{m} 1$ and receiving $\mathrm{m} 2$ at instance PCO is restricted to be between 8 and 10 seconds. The value of a time constraint is specified using 
intervals. The interval boundaries may be open, by using parenthesis, or closed, by using square bracket. An omitted lower bound is treated as zero, an omitted upper bound as infinite.

Time constraints can also be attached to the beginning and end of an inline expression (Figures 4 and 5). In this case, the constraint refers to the first or last event respectively which occurs inside the inline expression.

In addition to such relative time constraints, Fig. 7 contains a time constraint for a cyclic event (sending message $\mathrm{m} 1$ ) every $\bar{t}$ seconds) inside a loop inline expression. The definition of such periodic events is not supported in the MSC standard. Therefore, we use an extension proposed in [14].

\section{$2.2 \quad$ RTC-Patterns and MSC}

In the following, MSCs are used to present RTC-patterns for the most common hard real-time requirements [19010] 2 Since real-time requirements are always related to some functional behavior on which they are imposed, it is not possible to provide patterns for pure real-time requirements. Therefore, the RTC-patterns contain communication events on which the real-time requirements are imposed.

In order to ease specification and testing of real-time communication systems, it was our intention to provide patterns for testable real-time requirements only. In general, testable requirements can be obtained if the involved events of the system can be observed and stimulated. Thus, we assume that the system for which the requirements are specified has appropriate interfaces called points of control and observation (PCOs).

In our RTC-patterns, we represent each PCO as one MSC instance. The system is described by a single decomposed instance with the name System. We abstract from the internal structure of the system by omitting in the System instance header the actual reference to an MSC that refines the system behavior. Hence, we obtain a black-box view of the system.

The most common real-time requirements are related to delay, throughput, periodic events and jitter respectively. Basically, those requirements describe time relations between one sending and one receiving event, or the repeated occurrence of one sending and one receiving event. Depending on the number of PCOs of a system, the RTC-pattern for a certain requirement may look different, i.e., several pattern variants may exist for describing the same real-time requirement in different system configurations. In this paper, we provide RTC-patterns for systems with one or two PCOs only.

Delays: Latency/Response Time. The term delay is often used as an umbrella term for both latency and response time [9], since both only differ in the number of PCOs which are involved in the requirement. Hence, patterns for both types of real-time requirements are given.

${ }^{2}$ Note, that MSC is not well suited for expressing requirements involving statistical properties like soft real-time requirements or loss distributions. 


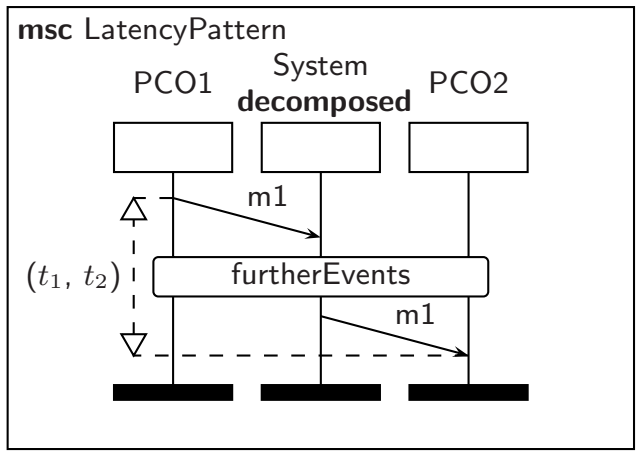

Fig. 2. Latency pattern

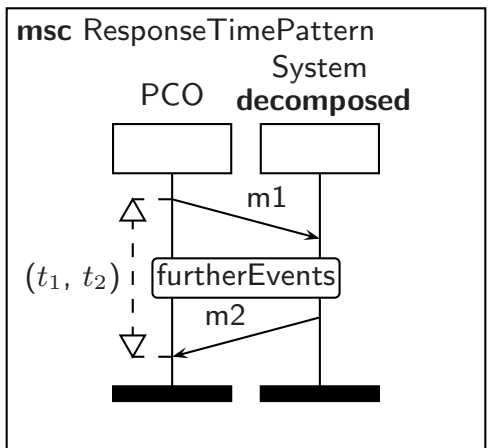

(a) Response time pattern

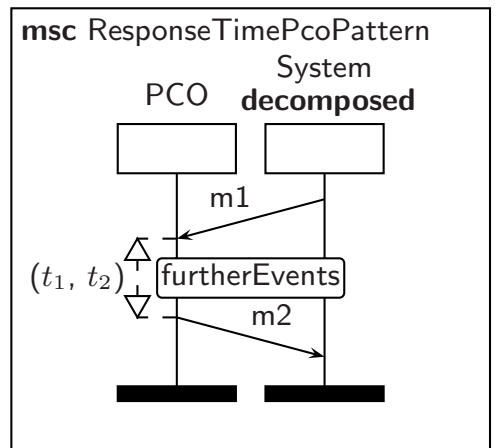

(b) Response time for the PCO pattern

Fig. 3. Response Time Patterns

Latency describes the delay which is introduced during the transmission of a signal by a component (the system), which is responsible for forwarding this signal [10]. The RTC-pattern for the latency requirement is given by the MSC LatencyPattern in Fig. 2, The allowed latency between sending message $\mathrm{m} 1$ via PCO1 and receiving it at PCO2 should be between $t_{1}$ and $t_{2}$ time units. The delay may be introduced by some further events that may include communication with the system environment (indicated by the MSC reference furtherEvents), the transmission times for message $\mathrm{m} 1 \mathrm{3}^{3}$, and additional computations inside the system (indicated by the decomposed keyword in the heading of the System instance).

Response time is a delay requirement where the same PCO is used for sending a message and receiving the corresponding answer. The response time pattern is shown in Fig. 3h. In contrast to the latency pattern, the messages in the response time pattern usually differ significantly, e.g., request (message m1) and response

${ }^{3}$ Even though in this pattern the same message name is used for both transmissions, the actual contents of the forwarded message may differ due to changes introduced by the system, e.g., updated hop counters or processing of the actual payload. 


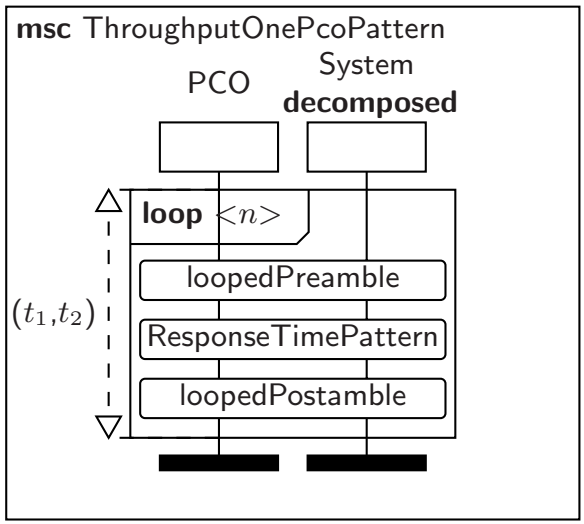

Fig. 4. Throughput pattern with one PCO

(message $\mathrm{m} 2$ ) in a client-server system. The given MSC shows a pattern for a response-time of $t_{1}$ and $t_{2}$ time units between sending message $\mathrm{m} 1$ and receiving message $\mathrm{m} 2$.

The response time requirement can also be turned into an requirement or assumption for the system environment or tester. This is necessary, if a timely behavior of the environment is needed by the system to fulfill some other requirements. This requirement can be specified using the response time PCO pattern given in Fig. 3 p.

Throughput. While delay-based real-time requirements focus on a systems performance for a single set of events, throughput requirements consider a systems performance over a longer duration. This means, the number of messages per time that a system has to deliver or to process repeatedly is constrained [9]. In MSC, this can be expressed using loop inline expressions with time constraints.

The throughput one PCO pattern shown in Fig. 4 captures a throughput requirement for communication which is observed at one PCO.

The loop inline expression includes the references loopedPreamble, ResponseTimePattern and loopedPostamble. ResponseTimePattern refers to RTC-patterns response time (Fig. 3a) or response time PCO (Fig. 3b). The response time patterns define the functional behavior, which is part of the throughput requirement. Additional behavior, which precedes or follows the response pattern, may be contained in the MSC references loopedPreamble and loopedPostamble.

Even if a throughput requirement is fulfilled, this does not necessarily imply that all response time requirements are fulfilled for each of the loop's iteration (e.g., due to bursty behavior and buffers inside the system). Thus, when inserting a response time pattern into the throughput pattern, it has to be considered whether only the functional behavior of a response time pattern is desired or also an additional real-time constraint. In the first case, the delay pattern has to be instantiated with the time interval $[0, \infty)$ which is equivalent to removing 


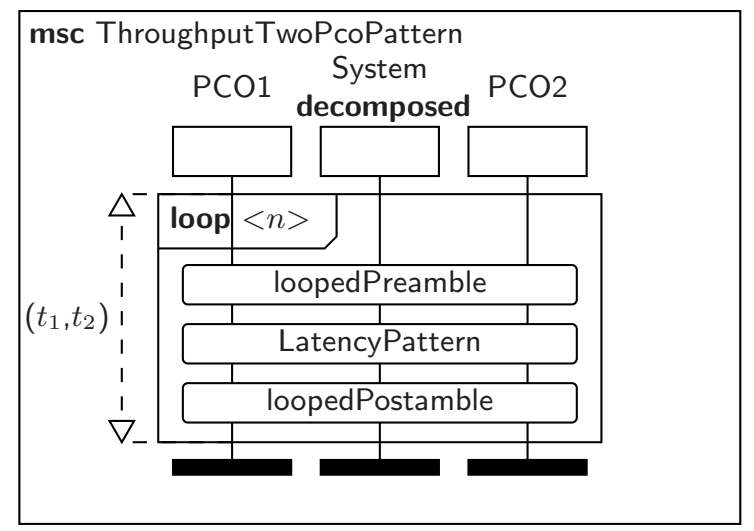

Fig. 5. Throughput pattern with two PCOs

the real-time constraint from the response time pattern. The latter case leads to requirements for periodic events and their jitter (see next section).

The given throughput pattern constrains a throughput TP to be $\frac{n}{t_{2}}<T P<$ $\frac{n}{t_{1}}$ events per time unit. Note, that those "events" typically consist of a set of events, in particular such according to one of the delay patterns presented before.

For specifying a throughput requirement, which is observed at two PCOs, the throughput two PCO pattern shown in Fig. 5 is appropriate. This RTC-pattern re-uses the latency pattern (Fig. 2) for describing the functional behavior, which is part of the throughput requirement.

Periodic Events and Jitter. In contrast to throughput requirements, requirements for periodic events have to hold for each single execution of a periodic event. Like for the throughput requirement, iteration of events can be obtained using MSC loop inline expressions — but for periodic requirements, the time constraint is contained inside the loop. Depending on the numbers of involved PCOs, several patterns are possible. In this paper, we can only present some selected cases.

The first class of periodic requirements can be obtained, if delay patterns are put inside the loop. As an example, Fig. 6 shows a cyclic response time pattern, where the response time pattern from Fig. 3 a has been chosen as delay pattern. Thus, the expressed real-time requirement is that the response time needs to hold every iteration of the loop.

Such MSCs can also be interpreted as delay jitter specifications. Delay jitter describes the variation of the delay during repetition. Note, that several interpretations of "jitter" exist [11]. Here, we use the following definition: $J_{i}=D_{i}-\bar{D}$, where $\bar{D}$ is the ideal (target) delay, $D_{i}$ the actual delay of the $i^{\text {th }}$ pair of events and thus $J_{i}$ the jitter in the $i^{\text {th }}$ repetition. Hence, a delay jitter requirement for the overall sequence of delays is expressed by the following inequation: $\forall i: J^{-}<J_{i}<J^{+}$, where $J^{-}$is the maximal allowed deviation below and $J^{+}$the maximal allowed deviation above the target delay $\bar{D}$. 


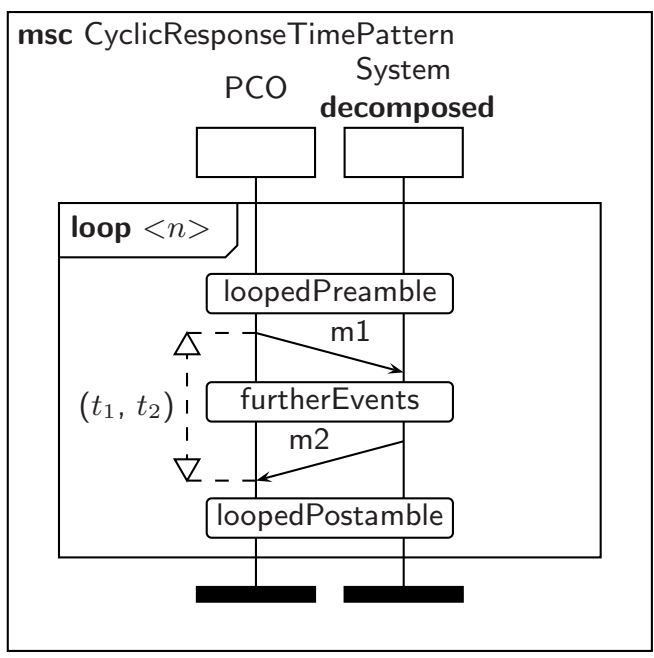

Fig. 6. Expansion of a looped response time pattern

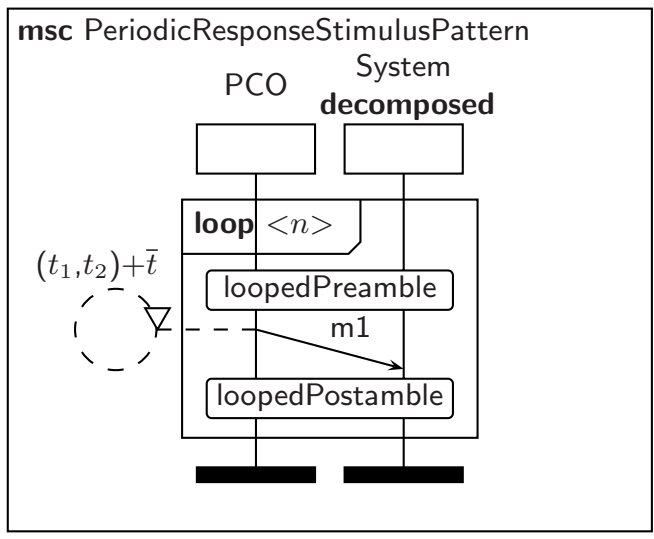

Fig. 7. Periodic response stimulus pattern

The RTC-pattern in Fig. 6 expresses a target delay $\bar{D}$ for which $t_{1}<\bar{D}<t_{2}$ holds and a delay jitter requirement with $J^{-}=t_{1}-\bar{D}$ and $J^{+}=t_{2}-\bar{D}$. I.e., the interval $\left(t_{1}, t_{2}\right)$ could alternatively be written as $\left(\bar{D}+J^{-}, \bar{D}+J^{+}\right)$.

While time constraints for delays can be easily expressed using MSC, it is not possible to express the periodicity of cyclic events, i.e., a frequency. The reason is, that standard MSC does not allow to attach time constraints to a pair of events which spans over adjacent repetitions of a loop. Thus, MSC extensions for either high-level MSC [15] or plain MSC [14] have been suggested. The notation for the extension of plain MSC is shown in Fig. 7. The semantics of this extension can be obtained by unrolling that loop as shown in Fig. 8 .

The periodic response stimulus pattern in Fig. [7specifies a periodic sending of message $\mathrm{m} 1$ to the system. The requested periodicity $\bar{t}$ is specified as an 


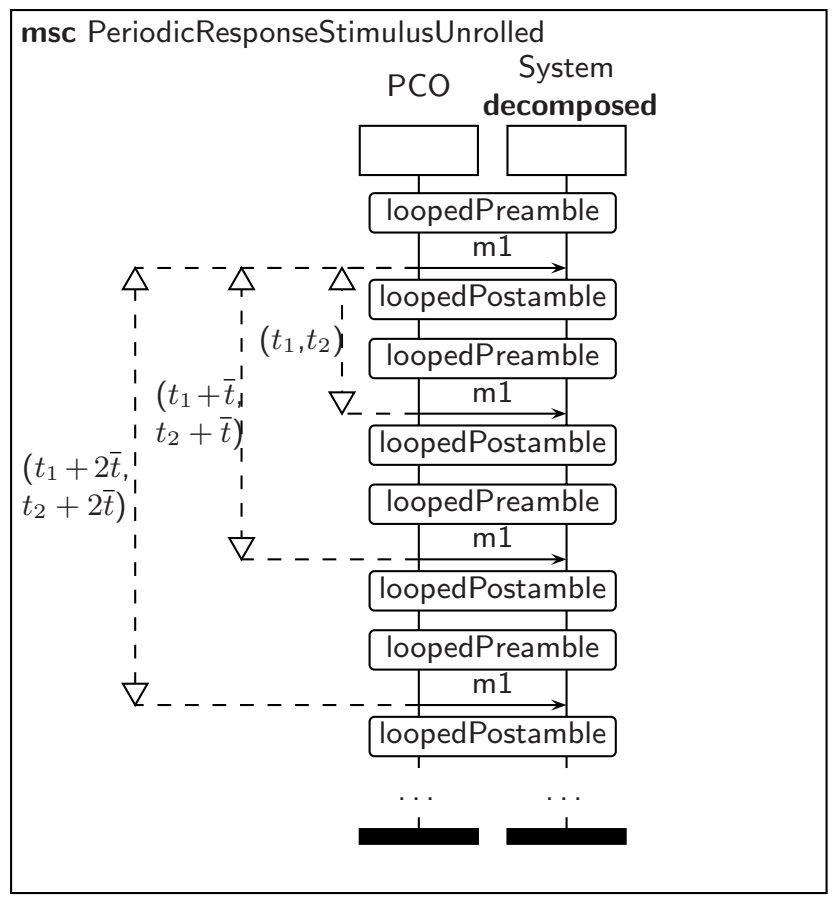

Fig. 8. Pattern of Fig. 7 with unrolled loop

additional parameter of the time interval. Likewise to delay jitter, a jitter for the periodicity or frequency jitter respectively is also specified by this pattern via $t 1$ and $t 2$, i.e. periodicity jitter requirement with $J^{-}=t_{1}-\bar{t}$ and $J^{+}=t_{2}-\bar{t}$.

Further patterns can be obtained if two PCOs are used or the periodicity constraint is attached to another event, e.g., if the frequency of a message reception at a PCO should be constrained.

\section{Application to Testing}

In the previous section, it was shown how MSC RTC-patterns can be applied for specifying real-time requirements. In this section, we demonstrate how the RTC-patterns can be used for test development with TIMEDTTCN-3. First, we describe how to associate RTC-pattern to TIMEDTTCN-3. Then, we provide an application of this approach using an example.

\subsection{Applying RTC-Patterns to TIMEDTTCN-3}

TIMEDTTCN-3 [3] is a real-time extension for TTCN-3 [5]. It introduces the concept of absolute time, extends the TTCN-3 logging mechanism, supports online and offline evaluation of tests and adds the new test verdict conf to the existing TTCN-3 test verdicts. 
(1) $\operatorname{var}$ float timeA, timeB;

(2) timeA := self.now;

(3) PCO1.send (m1);

(4) furtherEvents();

(5) PCO2.receive (m1);

(6) timeB := self.now;

(7) setverdict(evalLatencyOnline(timeA, timeB, t1, t2)); ...

Fig. 9. TIMEDTTCN-3 Code for online latency evaluation

This section does not introduce the TIMEDTTCN-3 language in detail. However, the presented TIMEDTTCN-3 code should be understandable for readers with some basic knowledge of common programing languages like, e.g., $\mathrm{C}++$. Further details about TTCN-3 and TIMEDTTCN-3 can be found in [3] and [5].

TIMEDTTCN-3 distinguishes between two different evaluation mechanisms for real-time requirements. On the one hand, online evaluation refers to the evaluation of a real-time requirement during the test run. On the other hand, offline evaluation means to evaluate a real-time requirement after the test run. We explain both by presenting the online evaluation of a latency requirement and by describing the offline evaluation of a throughput requirement.

Fig. 9 shows the TIMEDTTCN-3 code fragment, which is related to the latency RTC-pattern. The relevant events for measuring the latency of two events are the sending of message $\mathrm{m} 1$ and receiving of message $\mathrm{m} 1$ (cf. Fig. 2). Thus, before $\mathrm{m} 1$ is sent to the SUT and after $\mathrm{m} 1$ is received, the points in time are measured and stored in the variables timeA and timeB (lines 2 and 6 of Fig. 9). The online evaluation function for latency is called in Line 7 with the parameters of the measured time values, i.e., timeA and timeB, and the allowed timebounds which are supposed to be stored in $\mathrm{t} 1$ and $\mathrm{t} 2$.

The definition of the function evalLatencyOnline can be found in the lines 6-15 of Fig. 11. Fig. 11 is an excerpt of the library module EvaluationFunctionModule, which embodies all functions for real-time evaluations.

In Fig. 9] function evalLatencyOnline is called in Line 7 within a setverdict operation. Depending on the time measurement, the function returns a pass verdict, if the real-time requirement is met, or a conf verdict (=non-functional fail) if the requirement is not met. The setverdict operation sets the verdict of the test case to the result of evalLatencyOnline.

Lines 1-10 in Fig. 10] depict a code fragment for a test case developed with the throughput two PCO pattern (cf. Fig. 5) that uses the offline evaluation mechanism for the throughput requirement. The events relevant for throughput are executed in a loop. Since for throughput only the overall duration is of interest, only the time points immediately before and after the execution of the 


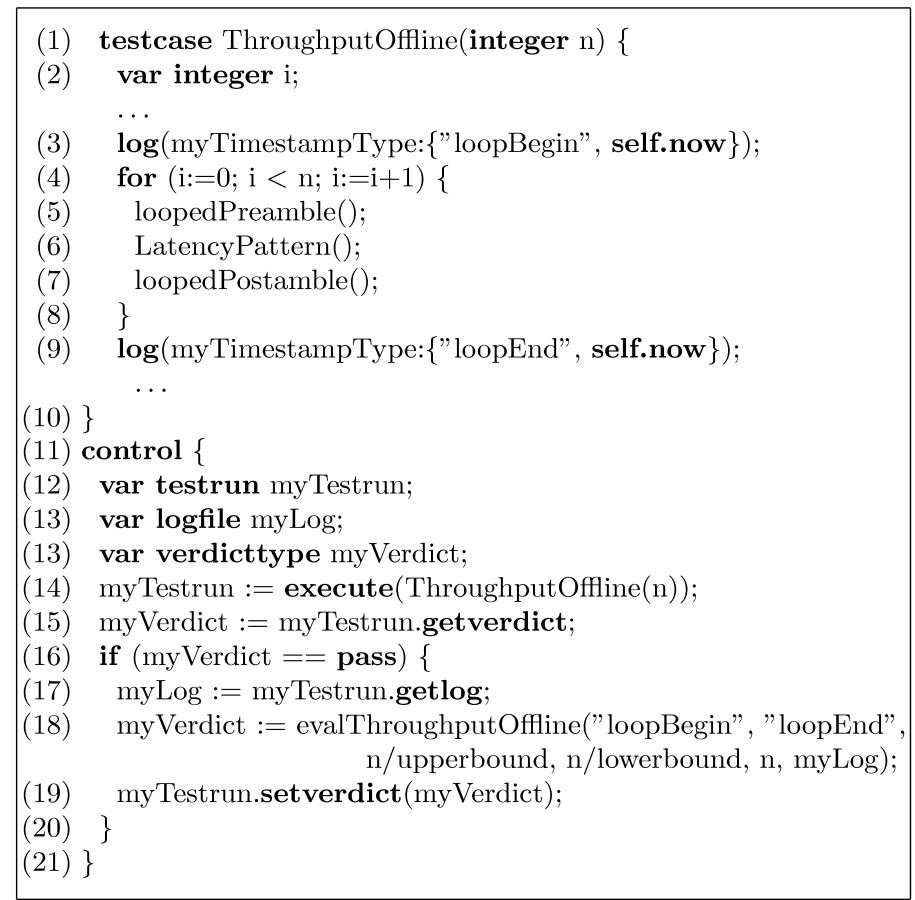

Fig. 10. TIMEDTTCN-3 Code for offline throughput evaluation

loop construct are measured and stored in a logfile (lines 3 and 9 of Fig. 10). Each entry of the logfile contains the name of the event and the associated time value, which is gained by the self.now statement.

In order to perform the offline evaluation, first test case ThroughputOffline is invoked in the control part of the TIMEDTTCN-3 module (Line 14 of Fig.10) and afterwards, the verdict of the functional behavior is checked (lines 15 and 16). If the functional verdict is a pass verdict, the real-time requirement will be evaluated. For that, the logfile is retrieved (Line 17) and the evaluation function evalThroughputOffline is called (Line 18). The parameters of the function are the identifiers of the logfile entries, the upper and lower throughput bound 4 , the number of iterations and the logfile generated by the test case.

The definition of function evalThroughputOffline can also be found in the library module EvaluationFunctionModule (lines 16-40 of Fig. 11). The function has six parameters: the labels of the entry and exit time stamps of the loop (loopEntry, loopExit), the lower and upper throughput bounds (lowerThroughput, upperThroughput), the number of iterations ( $\mathrm{n}$ ) and the logfile to evaluate (timelog). Lines 19-32 navigate to the relevant time stamps in the logfile and retrieve the entries: The operation first (Line 19) sorts the logfile entries and moves a cursor to the first

4 The throughput bounds are calculated from the number of iterations and the interval bounds. 


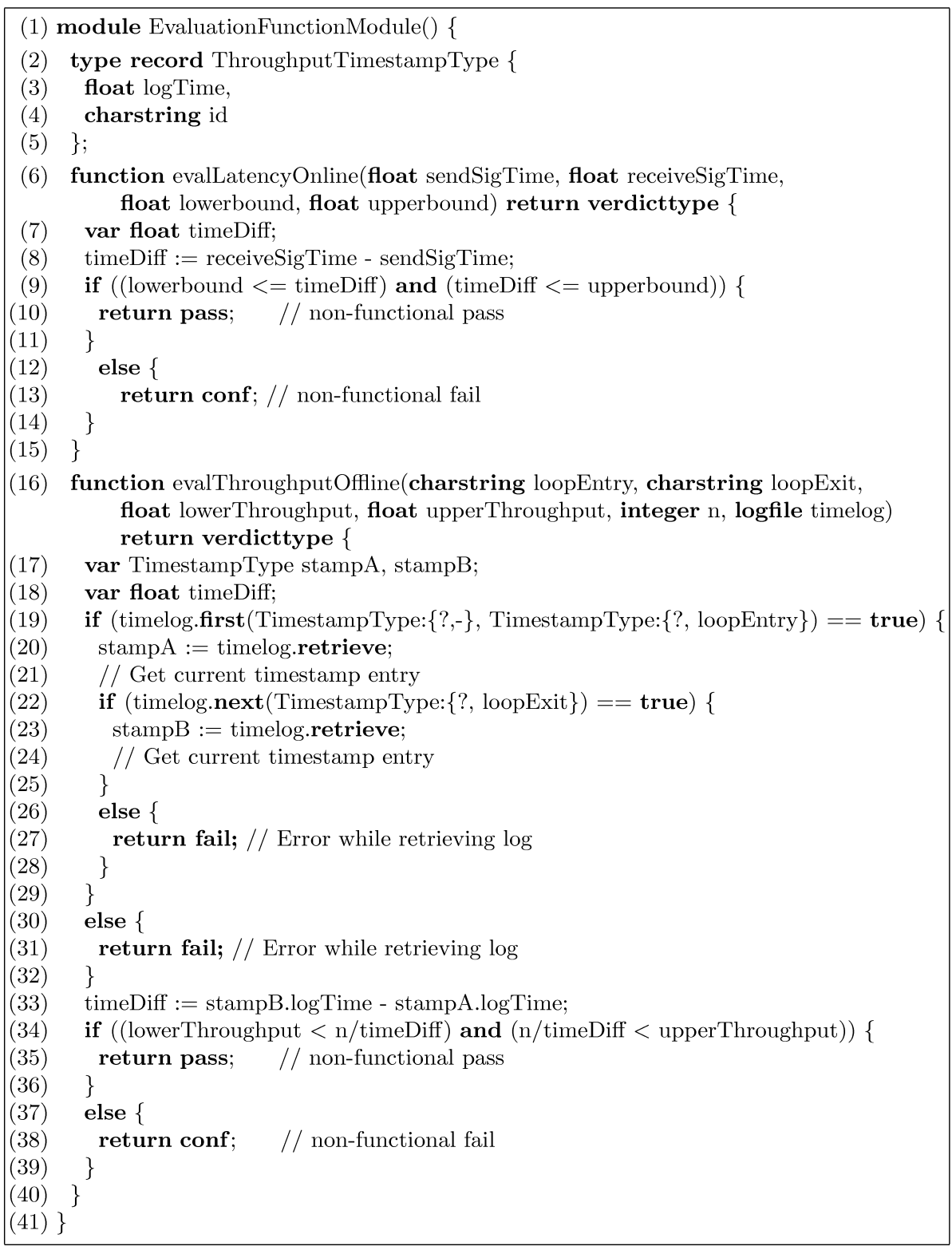

Fig. 11. Module with evaluation functions

matching entry in the logfile. A "?" indicates the field that is used as a sorting key. The second parameter of the first operation is used to move the cursor to the entry which relates to the loopEntry. The logfile entry which matches, is extracted by the retrieve operation (Line 20). The operation next (Line 22) advances the 


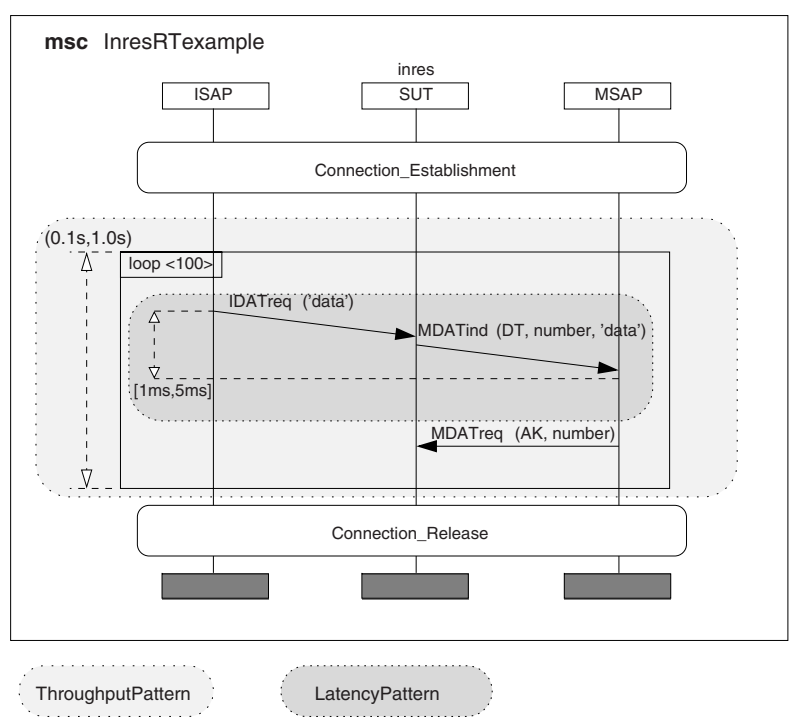

Fig. 12. Test purpose for the Inres example

cursor to the subsequent time stamp with a label identified by loopExit. The calculation of the actual throughput value is performed in lines 33-39 based on the arithmetic expression for throughput presented in Section 2.2 Depending on the evaluation, the function returns a pass verdict, if the real-time requirement is met, or a conf verdict if the requirement is violated.

In Fig. 10, the offline evaluation function is called in Line 18. The result of the function call is then used to set the final verdict of the test case (Line 19).

\subsection{The Inres Example}

Fig. [12 shows an MSC test purpose for testing an Initiator implementation of the Inres protocol [8] with real-time requirements. The Inres system can be accessed via the PCOs ISAP and MSAP. The functional requirement of the test purpose is to test 100 data transfers. For doing this, a connection needs to be established. After the test, the connection has to be released. The real-time requirements of the test purpose are to test:

1. a latency constraint between the signals IDATreq and MDATind, and

2. a throughput constraint on the loop construct.

When scanning through the given MSC diagram, the RTC-pattern for latency and throughput with two PCOs (Section 2.2) can be recognized. The shaded areas in Fig. 12 show where both patterns are located in the diagram.

In this example, the latency between IDISreq and MDATind shall be evaluated during the test execution (i.e., online) and the throughput of the loop construct after the test execution (i.e., offline). The MSC diagram does not define which 


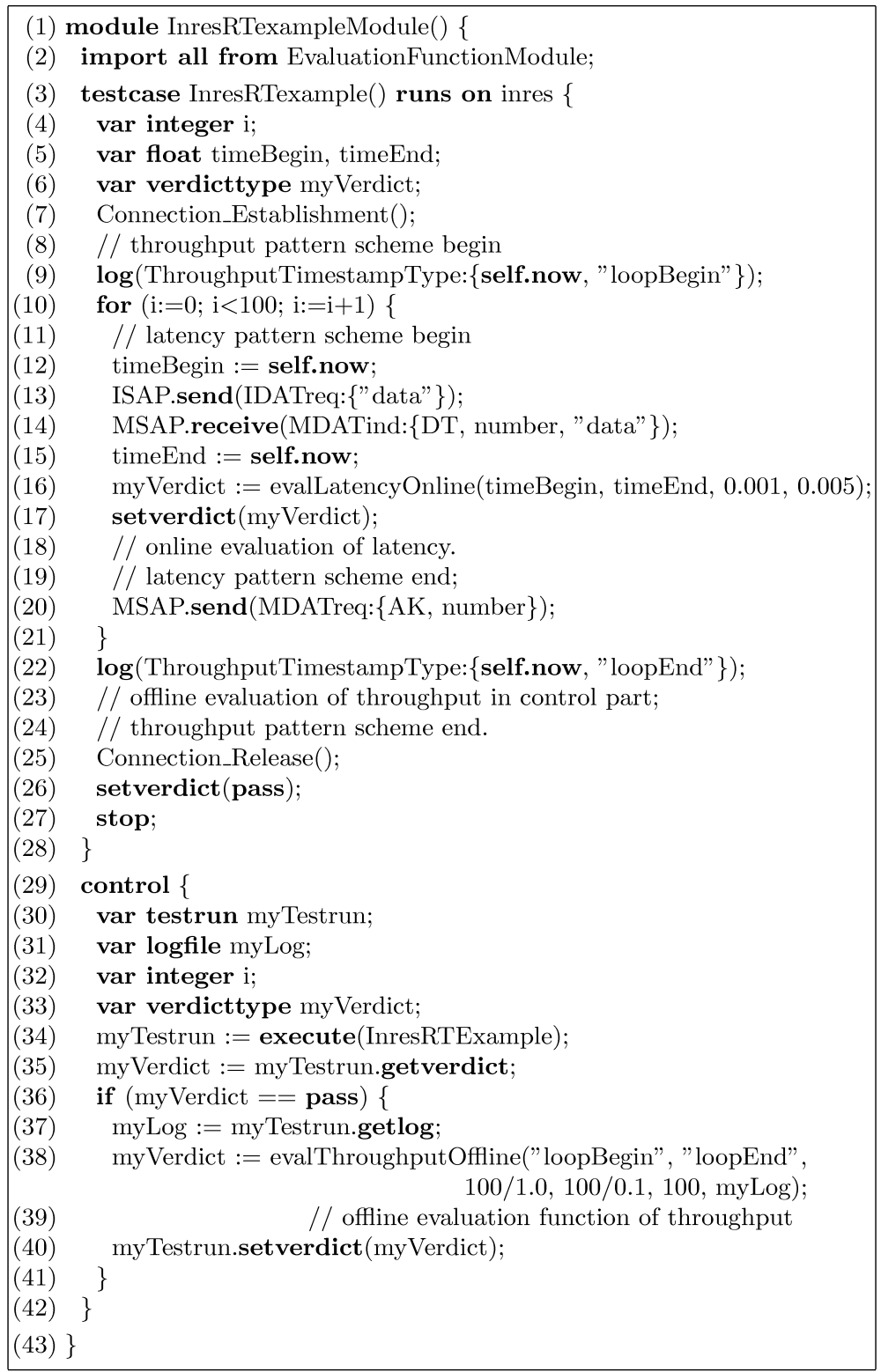

Fig. 13. Test case generated from Fig. 12

evaluation mechanism is desired since the MSC language does not provide the possibility to express those kind of requirements. We consider such information as directives for a code generation algorithm. 
In the previous section, we have introduced the TIMEDTTCN-3 code fragments and evaluation functions for online latency and offline throughput requirements (Figures 9, 10 and 11). Now, we shall utilize them in our example.

Fig. 13 shows the TIMEDTTCN-3 code for the Inres example, which can be generated automatically from the MSC diagram in Fig. 12. The module InresRTexampleModule (Fig. 13) imports all evaluation functions and types from the library EvaluationFunctionModule (Line 2). It contains only one test case called InresRTexample (lines 3-28) and the module control part (lines 29-42).

Test case InresRTexample starts with a connection establishment (Line 7). After connection establishment, points in time for the throughput measurement are logged. Analogous to the throughput code fragment, the time value and the event names are stored in the logfile just before the for loop contruct starts and just after it terminates (lines 9 and 22 in Fig. 13). These logged informations are accessed after the test run for the offline evaluation of the throughput requirement.

The online evaluation of latency between the signals IDATind and MDATreq is executed within the test case in lines 12-16. According to the code fragment presented in Section 3.1 the time before and after the time-critical events IDATreq and MDATreq are stored in the variables timeBegin and timeEnd. The evaluation is performed during the test run (Line 16) and the verdict of the test case is set in Line 17.

In the control part (lines 29-42), the throughput evaluation function is invoked. After the test case InresRTExample has been successfully executed regarding its functional behavior (lines 34-36), the logfile is fetched (Line 37) and the offline evaluation function evalThroughputOffline is called (Line 38). The final verdict is set with respect to the outcome of the evaluation function (Line 40).

\section{Summary and Outlook}

In this paper, MSC-based RTC-patterns for the specification of delay, throughput and periodic real-time requirements of communication systems have been presented. We demonstrated, how test development is eased, since pre-defined TIMEDTTCN-3 evaluation functions can be associated to each RTC-pattern.

RTC-patterns may also improve the requirements definition and the specification phase of an integrated development methodology for real-time communication systems. For this, the formalisation of instantiation and composition of MSC-based RTC-patterns has to be studied. A formalization is possible due to the formality of MSC. Further investigations on the required MSC extensions, tool support and the usability of such an approach is necessary. Such investigations will be the focus of our future work.

Furthermore, we will implement support for RTC-patterns in our tool, which translates MSC test descriptions into TIMEDTTCN-3 test cases. This includes also the provision of a library of generic evaluation functions for the RTC-patterns. 


\section{References}

1. ATM Forum Performance Testing Specification (AF-TEST-TM-0131.000). The ATM Forum Technical Committee, 1999.

2. F. Buschmann, R. Meunier, H. Rohnert, P. Sommerlad, and M. Stal. PatternOriented Software Architecture - A System of Patterns. Wiley, 1996.

3. Z.R. Dai, J. Grabowski, and H. Neukirchen. TIMEDTTCN-3 - A Real-Time Extension for TTCN-3. In I. Schieferdecker, H. König, and A. Wolisz, editors, Testing of Communicating Systems, volume 14, Berlin, March 2002. Kluwer.

4. Z.R. Dai, J. Grabowski, and H. Neukirchen. TIMEDTTCN-3 Based Graphical Real-Time Test Specification. In D. Hogrefe and A. Wiles, editors, Testing of Communicating Systems, volume 2644 of Lecture Notes in Computer Science (LNCS). Springer, May 2003.

5. ETSI European Standard (ES) 201 873-1 (2002). The Testing and Test Control Notation version 3; Part 1: TTCN-3 Core Language. European Telecommunications Standards Institute (ETSI), Sophia-Antipolis (France), also published as ITU-T Rec. Z.140.

6. E. Gamma, R. Helm, R. Johnson, and J. Vlissides. Design Patterns - Elements of Reusable Object-Oriented Software. Addison Wesley, 1995.

7. B. Geppert. The SDL Pattern Approach - A Reuse-Driven SDL Methodology for Designing Communication Software Systems. PhD thesis, University of Kaiserslautern (Germany), July 2001.

8. D. Hogrefe. Report on the Validation of the Inres System. Technical Report IAM-95-007, Universität Bern, November 1995.

9. Request for Comments 1193: Client requirements for real-time communication services. Internet Engineering Task Force (IETF), 1990.

10. Request for Comments 1242: Benchmarking Terminology for Network Interconnection Devices. Internet Engineering Task Force (IETF), July 1991.

11. Request for Comments 3393: IP Packet Delay Varation Metric for IP Performance Metrics (IPPM). Internet Engineering Task Force (IETF), November 2002.

12. ITU-T Rec. Z.100 (1999). Specification and Description Language (SDL). International Telecommunication Union (ITU-T), Geneve.

13. ITU-T Rec. Z.120 (1999). Message Sequence Chart (MSC). International Telecommunication Union (ITU-T), Geneve.

14. H. Neukirchen. Corrections and extensions to Z.120, November 2000. Delayed Contribution No. 9 to ITU-T Study Group 10, Question 9.

15. T. Zheng and F. Khendek. An extension to MSC-2000 and its application. In Proceedings of the 3rd SAM (SDL and MSC) Workshop, 2002. 